\title{
La vision des pharmaciens sur le Chemotherapy Foundation Symposium Compte rendu du congrès organisé à New York (6-8 novembre 2013) *
}

\author{
Pharmacists' Point of View on the Conference Chemotherapy Foundation Symposium \\ Report Organized in New York (6-8 November 2013)
}

\author{
O. Aujoulat $\cdot$ F. Basuyau $\cdot$ N. Cormier $\cdot$ D. Dautel $\cdot$ F. De Crozals $\cdot$ M. Doly $\cdot$ M. Duban $\cdot$ B. Favier $\cdot$ I. Madelaine- \\ Chambrin - C. Ollivier · M. Paul - I. Princet $\cdot$ E. Serafini-Fougereau $\cdot$ F. Tabary $\cdot$ J.-F. Tournamille $\cdot$ J.-B. Rey
}

Reçu le 2 décembre 2013; accepté le 19 décembre 2013

(C) Les auteurs 2014. Cet article est publié en Open Access sur link.springer.com

Résumé Le Chemotherapy Foundation Symposium, le congrès de l'hôpital du Mount Sinaï, s'est tenu comme tous les ans à New York. Entre les congrès « de l'été » (ASCO et ESMO) et ceux « de l'hiver » (ASH, San Antonio Breast Cancer Symposium), la majorité des résultats 2013 déjà publiés ou annoncés sont regroupés à l'occasion de ce congrès généraliste d'oncologie. Pendant les quelques jours de congrès, des pharmaciens ont assisté aux communications et les rapportent ici, en les regroupant par organes (hématologie, cancers digestifs, cancers gynécologiques, cancers du sein, cancers urologiques et cancers du poumon, ORL et mélanomes).

\section{O. Aujoulat}

Service pharmacie, hôpital Emile-Muller,

Mulhouse, France

F. Basuyau

Service pharmacie, centre Henri-Becquerel, Rouen, France

\section{N. Cormier}

Service pharmacie, centre Catherine-de-Sienne, Nantes, France

\section{Dautel}

Service pharmacie, centre hospitalier de Valenciennes,

Valenciennes, France

F. De Crozals

Service pharmacie, institut Sainte-Catherine, Dijon, France

M. Doly

Département de pharmacie, centre Jean-Perrin,

Clermont-Ferrand, France

\section{Duban}

Service pharmacie, centre Georges-François-Leclerc,

Dijon, France

B. Favier

Service pharmacie, centre Léon-Bérard, Lyon, France
Mots clés Chemoterapy Foundation Symposium · Pharmaciens

Abstract Chemotherapy Foundation Symposium, the Congress of Mount Sinai Hospital, is held every year in New York. Between "summer" (ASCO and ESMO) and "winter" (ASH, San Antonio Breast Cancer Symposium) conferences, the majority of results already published or announced in 2013 were grouped on the occasion of this general oncology congress. During the few days of congress, pharmacists attended meetings and presented reports, grouped by organ (hematology, gastrointestinal cancers, gynecological

I. Madelaine-Chambrin

Service pharmacie, hôpital Saint-Louis, Paris, France

C. Ollivier

Service pharmacie, hôpital Clémenceau, Caen, France

M. Paul

Service pharmacie, hôpital Henri-Mondor, Créteil, France

I. Princet

Service pharmacie, CHR la Miletrie, Poitiers, France

E. Serafini-Fougereau

Service pharmacie, institut Paoli-Calmettes, Marseille, France

F. Tabary

Service pharmacie, GCS UPCM, Montpellier, France

J.-F. Tournamille

Service pharmacie, hôpital Bretonneux, Tours, France

J.-B. Rey $(\bowtie)$

Département de pharmacie, institut Jean-Godinot,

1, rue du Général Kœnig, F-51100 Reims, France

e-mail : jean-baptiste.rey@reims.unicancer.fr

* Article réalisé avec le soutien du laboratoire Roche. 
cancers, breast cancers, urological cancers and lung cancers, head and neck cancers, and melanoma).

Keywords Chemotherapy Foundation Symposium · Pharmacists

\section{Introduction}

Michel Doly

C'est devenu maintenant un repère dans « l'année pharmaco-oncologique » : la réunion organisée par le Mount Sinaï Hospital de New York sur les innovations dans la prise en charge thérapeutique des cancers. C'est une manifestation à laquelle participe un nombre limité de spécialistes mais qui reprend les données les plus récentes de chimiothérapie et de radiothérapie des cancers.

C'est devenu maintenant également « une tradition » que, grâce au laboratoire Roche, un groupe de pharmaciens hospitaliers participe à cette réunion, toujours de façon très active. Nous avons choisi, en effet, de « croiser nos regards » sur les différentes communications et d'échanger entre nous sur notre perception et notre compréhension de ces innovations.

C'est un exercice exigent mais très riche qui permet à chaque membre de ce groupe de bénéficier de l'expertise de chacun. La qualité de nos échanges nous a conduits à souhaiter une trace écrite de ce travail.

C'est l'objet de cet article, que nous cosignons, qui a été écrit par l'un d'entre nous sur la base de nos comptes rendus et de nos discussions. Il s'agit donc d'un travail collectif qui n'a été possible que grâce à une réelle complicité d'ailleurs partagée avec nos interlocuteurs du laboratoire Roche.

\section{Hématologie}

\section{Isabelle Madelaine-Chambrin, Catherine Ollivier, Muriel Paul}

Comme chaque année, le congrès était très riche en communications sur les hémopathies malignes. Outre les communications dédiées aux différentes pathologies, il est ressorti de ces sessions, un certain nombre d'informations sur les nouveaux traitements. Les premiers d'entre eux sont ceux qui ciblent les voies de signalisation, notamment les inhibiteurs de Bruton kinase et les inhibiteurs de PI3K, mais aussi l'obinutumumab (un nouvel anticorps monoclonal antiCD20) et un inhibiteur de FLT3 dans la prise en charge de la LAM. Le récepteur des cellules B (B cell receptor $[\mathrm{BCR}]$ ) active différentes protéines qui aboutissent à l'activation de la Bruton kinase qui active, elle-même, d'autres voies de signalisation, dont la voie de la PI3-kinase.

\section{Nouvelles molécules}

Le premier traitement dont nous rapporterons les données ici est l'ibrutinib (PCI-32765). C'est un inhibiteur oral de la Bruton kinase, indiqué dans les leucémies lymphö̈des chroniques (LLC) ; il se fixe de façon irréversible sur la Bruton kinase (à noter que des inhibiteurs réversibles sont aussi en cours de développement). Il peut être associé aux thérapeutiques dirigées contre le $\mathrm{CD} 20$, avec des réponses objectives très importantes. Dans les différentes études de développement, les doses utilisées ont été assez variables avec une difficulté à déterminer la dose efficace. En revanche, pour l'ibrutinib, il a été montré une relation AUC-dose, linéaire avec une saturation des sites de la Bruton kinase en fonction de l'AUC. Il a été montré que l'AUC de $160 \mathrm{mg} . \mathrm{H} / \mathrm{ml}$ permet une saturation complète ; la dose optimale dépendrait donc de cette valeur cible. À noter qu'en termes de relation PK-PD, il y a peu de thérapies ciblées pour lesquelles ce type de relation a pu être mise en évidence.

En termes de tolérance, l'ibrutinib se caractérise par des diarrhées importantes d'apparition parfois très rapide (dès la première prise), pouvant conduire à des déshydratations majeures, accompagnées d'hyponatrémie ; parmi les autres effets indésirables observés, des patients ont présenté des nausées-vomissements, des épisodes d'hypertension artérielle, ainsi que des pneumonies infectieuses dont il est difficile de déterminer si elles sont dues au produit ou si elles sont une complication de la LLC. Le dossier d'enregistrement de ce produit est déjà en cours dans les LLC, les lymphomes à petites cellules, mais aussi les lymphomes du manteau. Dans la LLC, l'efficacité se traduit par une fonte spectaculaire des ganglions, mais aussi par une lymphocytose initiale, en raison d'un passage des lymphocytes de la moelle vers le sang, suivie d'une diminution progressive au cours de la première année.

L'ibrutinib a été étudié dans le cadre d'une étude de phase II, chez des patients âgés de plus de 65 ans et présentant une LLC. Les résultats ont montré un taux de réponse similaire chez des patients naïs et chez des patients en rechute; cependant, la survie sans progression était de $96 \%$ à 26 mois chez les patients naïfs alors qu'elle n'était « que » de $74 \%$ chez les patients en rechute. Dans l'analyse en sous-groupes, les patients qui présentaient une délétion 17p (LLC de mauvais pronostic et donc des patients en impasse thérapeutique), le taux de réponse objective a été de $60 \%$, ce qui rend l'ibrutinib « intéressant» pour cette catégorie de patients. L'ibrutinib sera prochainement disponible en ATU (probablement début 2014) ; son enregistrement est en cours dans le cadre d'une procédure accélérée de la FDA et d'autres essais sont en cours, notamment en association avec le rituximab.

Par ailleurs, le développement se poursuit dans le cadre des lymphomes non hodgkiniens indolents et réfractaires 
(phase I) ; dans cette indication, le taux de réponse objective est de l'ordre de $60 \%$, avec $16 \%$ de réponse complète et une survie sans progression médiane de 13,6 mois ; il est à noter que, au cours de cette étude de phase I, la dose maximale tolérée n'a pas été atteinte. De même, dans une étude de phase II chez des patients atteints de lymphome diffus à grandes cellules, les résultats montrent des taux de réponse très prometteurs avec $40 \%$ de réponse chez les patients naïfs ( $17 \%$ de réponse complète) et une survie médiane de dix mois.

Le second produit sur lequel nous nous attarderons est l'idelalisib (CAL 101) ; c'est un inhibiteur de PI3K delta. C'est une thérapie orale, administrée à la dose de $300 \mathrm{mg}$ répartis en deux doses quotidiennes. Le profil de tolérance est un peu similaire à celui de l'ibrutinib avec des réactions à types de diarrhées, fièvre, rash cutané, mais aussi une élévation des enzymes hépatiques qui nécessitent une surveillance particulière. Ce produit présente également un intérêt en association aux cytotoxiques et aux thérapeutiques ciblant le CD20. L'idelalisib a été évalué dans une étude de phase II chez des patients atteints de LLC. Comme pour l'ibrutinib, chez des patients de profil similaire, les résultats montrent $80 \%$ de réponse chez des patients en rechute, une survie sans progression médiane de 17 mois et, tout comme l'ibrutinib, ce produit montre un intérêt chez les patients qui présentent une délétion $17 \mathrm{p}$. Lorsqu'il est associé au rituximab ou à l'ofatumumab, les résultats sont, là encore, impressionnants avec (respectivement) des taux de réponse objective de 90 et $94 \%$ et un taux de réponse complète à deux mois de $20 \%$ pour le bras en association au rituximab.

Il est à noter que l'étude GS 116, comparant rituximab seul à idelalisib associé au rituximab chez des patients présentant une LLC en rechute (modalité thérapeutique américaine, non retrouvée en Europe), a été arrêtée prématurément en raison des très bons résultats de survie sans progression, en faveur de l'association. D'ailleurs, l'enregistrement auprès de la FDA a été déposé et l'autorisation de mise sur le marché devrait intervenir tout aussi rapidement que pour l'ibrutinib.

En somme, deux AMM vont être accordées quasi simultanément à deux produits très efficaces dans une même indication.

En outre, comme pour l'ibrutinib, l'idelalisib a été évalué dans les lymphomes non hodgkiniens indolents, et les résultats de ces études ont également été bons, avec $80 \%$ de patients répondeurs et une survie sans progression de 17 mois environ.

Outre ces «nouveautés », le lénalidomide a été le sujet de nombreuses présentations lors du congrès. Le lénalidomide est un immunomodulateur cytotoxique qui interagit avec le microenvironnement de la tumeur. La première étude présentée était un essai de phase II chez des patients prétraités par le protocole FCR, associant ofatumumab et lénalidomide
$(10 \mathrm{mg} / \mathrm{j}$ en continu à partir du neuvième jour suivant l'administration de l'ofatumumab) ; les résultats ont montré un taux de réponse objective de près de $70 \%$ (taux de réponse complète $=24 \%$ ), mais aussi une toxicité hématologique limitante $(47 \%$ des patients ont présenté des toxicités de grade 4). D'autres études, de phase II et de phase III, plus positives ont également été présentées, notamment chez des patients présentant des lymphomes non hodgkiniens réfractaires avec de bons taux de réponse dans tous les sous-types cytologiques (réponse objective de l'ordre de $50 \%$ ).

Par ailleurs, l'étude R2-CHOP en première ligne chez des patients avec des lymphomes non hodgkiniens agressifs, associant lénalidomide $(25 \mathrm{mg} / \mathrm{j})$, rituximab et CHOP tous les 21 jours, a montré un taux de réponse objective de $98 \%$ avec $83 \%$ de réponse complète ; cependant, ce taux de réponse exceptionnel était associé à une toxicité hématologique majeure $(70 \%$ de patients présentant des toxicités de grade 4, nécessitant le recours aux G-CSF). Enfin, une nouvelle modalité thérapeutique a été présentée, sans faire intervenir de traitement cytotoxique, en associant uniquement le rituximab au lénalidomide ; cette étude américaine a montré des taux de réponse objective prometteurs de $90 \%$.

Parmi les anticorps anti-CD20 (rituximab, ofatumumab et obinutuzumab), c'est l'obinutuzumab (également connu sous le nom de GA 101) qui a retenu notre attention. C'est un anticorps anti-CD20 glycosylé dont l'ADCC est augmentée par rapport au rituximab. Il s'agissait d'une étude de phase III (étude CLL11, également présentée à l'ASCO 2013) comparant le rituximab au GA 101 associé au chloraminophène vs le chloraminophène seul chez des patients atteints de LLC. Les résultats ont montré des taux de réponse objective de $75 \%$ avec le GA 101, $66 \%$ avec le rituximab et de $30 \%$ dans le groupe témoin (chloraminophène qui, de fait, n'est plus un standard de traitement). C'est cette étude CLL11 qui constitue le dossier d'enregistrement du GA 101.

Le dernier produit présenté lors des sessions de ce congrès était le quizatinib (AC220) qui est un inhibiteur de FLT3, indiqué dans les leucémies aigües myéloïdes de mauvais pronostic du sujet jeune ou âgé. FLT3 est un récepteur membranaire à activité tyrosine-kinase qui est muté dans 20 à $25 \%$ des LAM. Cet inhibiteur de FLT3 provoque une différenciation cellulaire avec les blastes circulants qui sont mis en apoptose et les cellules de la moelle qui se différencient en cellules normales (polynucléaires neutrophiles) ; il s'agit donc d'un mécanisme d'action un peu original. Il est administré à raison de $200 \mathrm{mg} / \mathrm{j}$ et son profil de tolérance a montré un allongement de l'espace QT à surveiller en particulier, et des toxicités hématologiques. Les résultats des études cliniques ont montré qu'il était actif quel que soit l'âge des patients. Les taux de réponse sont respectivement de 46 et $57 \%$ chez les patients jeunes et les patients âgés. 
Cependant, il peut survenir des résistances au traitement assez rapidement, en 6 à 23 semaines.

Une partie des sessions dédiées à l'hématologie traitait des récepteurs $T$ artificiels encore appelés CARs pour chimeric antigen receptor. Ce récepteur combine à la fois les propriétés d'un anticorps et celles du récepteur $\mathrm{T}$ des lymphocytes. Ils ont été évalués dans certaines hémopathies à cellules B comme la LLC.

La partie extracellulaire du CAR est représentée par un anticorps simple chaine $(\mathrm{scFv})$ dirigé contre le récepteur CD19 ; ce dernier étant un récepteur exprimé à la surface des cellules B à partir du stade pré-B. Les parties transmembranaire et intracellulaire correspondent à l'unité de signali-

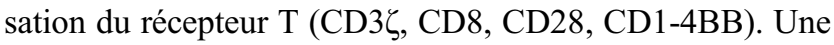
fois exprimé sur les lymphocytes $T$ après manipulation génétique, il se produit une réactivation des lymphocytes $\mathrm{T}$ cytotoxiques, et ce, indépendamment du CMH. Le CD19 présent sur le lymphocyte B est alors reconnu par le scFv de l'anticorps et il s'en suit une lyse des lymphocytes B.

Aujourd'hui, c'est la troisième génération de ces CARs qui est disponible :

Le traitement comprend plusieurs phases :

- aphérèse, quatre semaines avant le traitement permet de récupérer les lymphocytes $\mathrm{T}$ du patient ;

- manipulation génétique afin d'inclure dans le génome du virus la séquence codant pour le CAR ;

- mise en contact des lymphocytes $\mathrm{T}$ avec les virus transfectés ;

- incubation et phase de multiplication des LT modifiés ;

- chimiothérapie aplasiante chez le patient pendant le temps de manipulation : suppression des clones malins ;

- injection des lymphocytes T modifiés.

Il s'en suit une lyse majeure des lymphocytes B avec, parfois, un syndrome de lyse (risque de lymphopénie majeure chez les patients, même deux ans après le traitement).

Deux essais ont été présentés pendant le congrès, l'essai UPenn (huit patients en cinquième ligne de traitement pour certains) et l'essai MSKCC (quatre patients) en rechute après allogreffe, chez des patients avec une LLC ; les CARs injectés étaient différents dans les deux essais (deuxième génération pour l'essai MSKCC et troisième génération pour l'essai UPenn). Il y a eu, respectivement, trois réponses complètes et deux réponses complètes pour les essais UPenn et MSKCC respectivement. Il s'agit donc là de thérapies très prometteuses. À suivre...

\section{Lymphomes du manteau}

Un certain nombre de ces traitements sont évidemment retrouvés dans la prise en charge d'autres pathologies, à commencer par le lymphome du manteau. Cette pathologie est rare $(7 \%$ des $\mathrm{LNH})$ et c'est une maladie incurable en dehors de l'allogreffe de cellules souches. Elle touche principalement des hommes (sex-ratio $\mathrm{h} / \mathrm{f}: 3 / 1$ ), d'âge moyen de 58 ans, et elle est souvent découverte à un stade avancé (stade IV). Leur présentation est très variée (symptomatologie des lymphomes B, fièvre, sueurs nocturnes, perte de poids pas systématiquement retrouvée), ce qui rend leur diagnostic difficile. L'élément permettant d'affirmer le diagnostic est la translocation chromosomique $\mathrm{t}(11 ; 14)$ (q13;q32), qui juxtapose le gène CCND1 au gène codant pour la chaîne lourde des immunoglobulines, entraînant une expression anormalement élevée d'un régulateur du cycle cellulaire, la cycline D1, dans le noyau des cellules lymphomateuses ; d'un point de vue immunophénotypique, le lymphome du manteau se caractérise par un profil CD10-, CD23- et CD5+.

Classiquement, les premières lignes de traitement sont des associations de type R-CHOP, RHYPERCVAD, R$\mathrm{CHOP}+$ radiothérapie ou R-bendamustine ou des protocoles plus intensifs avec autogreffe pour les sujets jeunes. Ainsi, aux États-Unis, le schéma RHYPERCVAD + méthotrexate haute dose + autogreffe donne des taux de réponse de $97 \%$, mais s'accompagne d'un taux de décès toxiques très importants ; en Europe, le schéma R-CHOP alterné avec des protocoles R-DHAP suivi d'autogreffe donnent des taux de réponse identiques et est le traitement de référence actuel des patients de moins de 65 ans. Chez les patients âgés, c'est le standard de huit cures de R-CHOP qui donne les meilleurs résultats.

Après le traitement de première ligne, la rechute reste tôt ou tard inévitable. Le traitement de seconde ligne comporte des agents de chimiothérapie non encore utilisés lors du traitement de première ligne de type R-ICE ; R-ESHAP, R-B, RVB. De nouvelles options thérapeutiques sont proposées, notamment le bortezomib qui a une autorisation de mise sur le marché dans ce contexte aux États-Unis. Mais aussi la bendamustine (AMM en première ligne, mais pas en deuxième ligne, bien qu'utilisée en pratique dans ce contexte) ou le temsirolimus (AMM en rechute). Par ailleurs, des produits sont en développement dans cette indication, notamment l'ibrutinib et l'idelalisib (cf. supra), mais aussi, l'ABT 199 qui est un inhibiteur d'apoptose.

Lors du traitement d'un lymphome du manteau, si le patient est jeune (ou « fit »), l'objectif est d'obtenir la rémission la plus longue possible et d'améliorer ainsi la survie des patients. Pour les sujets asymptomatiques, se pose la question du choix entre l'abstention thérapeutique et le traitement intensif. En outre, ce traitement doit-il comporter les nouveaux produits non cytotoxiques ? C'est une question à laquelle chaque hématologue doit répondre pour ce type de patients, et c'est réellement une réponse au cas par cas qu'il faut donner. À l'inverse, chez les patients âgés, l'objectif est de contrôler la maladie, avec des toxicités moindres et une qualité de vie meilleure ; là encore, la même question de l'abstention thérapeutique se pose. En revanche, si le patient doit être traité, il faut se poser la question de l'option R- 
CHOP ou R-bendamustine, de l'intérêt de la maintenance par rituximab, mais aussi du traitement sans médicament cytotoxique (uniquement des thérapies ciblées).

L'essai présenté (EMERGE MCL 001) avec le lénalidomide était une étude de phase II en monothérapie, conduite chez 134 patients en rechute, en progression, ou présentant un lymphome du manteau réfractaire au bortezomib. Le lénalidomide était administré à la dose de $25 \mathrm{mg} / \mathrm{j}$ pendant 21 jours sur 28 jusqu'à progression de la maladie. Les taux de réponse observés étaient de l'ordre de $30 \%$ avec $8 \%$ de réponse complète dans tous les groupes de patients. La durée médiane de réponse était de 16,6 mois avec une survie sans progression de quatre mois et une survie globale de 19 mois. Ces résultats laissent penser que ce traitement pourrait présenter un intérêt dans ce cadre.

Par ailleurs l'ibrutinib, mais aussi l'idelalisib, le dasatinib, le fostamatinib (inhibiteur de Syk), l'ABT 199 et le palbococlib (inhibiteur de cycline) ont également été évalués. L'idelalisib a montré des taux de réponse globale de $40 \%$ en monothérapie (avec une survie sans progression de 3,7 mois) et des taux de réponse augmentés à $80 \%$ lorsqu'il est associé au rituximab, à la bendamustine ou aux deux (rituximab + bendamustine). Dans cet essai, le profil de tolérance n'a rien apporté de nouveau par rapport aux données déjà citées cidessus. Par ailleurs, l'essai Alliance A051201, concernant des patients en rechute et associant idelalisib, lénalidomide et rituximab est en cours et aucun résultat n'est encore disponible.

Enfin, l'ibrutinib administré à la dose de $560 \mathrm{mg}$ en continu par cycles de 28 jours jusqu'à progression était bien toléré avec $13 \%$ d'effets secondaires de grade 3 et $8 \%$ de grade 4 : toxicité principalement hématologique $(11 \%$ de neutropénie, $5 \%$ d'anémie et $5 \%$ de thrombopénie) ; plusieurs études sont encore en cours. Les premiers résultats d'une étude de phase II, incluant 111 patients ont montré des taux de réponse actualisés en 2012 de 74,5\% avec $35,3 \%$ de réponses complètes. La PFS et l'OS estimées à un an étaient de 53 et $67 \%$, respectivement. Ce suivi plus long confirme l'activité impressionnante de l'ibrutinib dans le lymphome du manteau en rechute/réfractaire, ainsi qu'un profil de toxicité favorable, ce qui a conduit à un enregistrement accéléré du produit par la FDA.

Des essais sont en cours chez des patients naïfs de traitement, en association avec bendamustine-rituximab (essai MCL3002) et chez des patients en rechute ou réfractaires où l'ibrutinib comparé au temsirolimus (MCL3001).

\section{Myélomes}

De pronostic aussi mauvais que le lymphome du manteau, le myélome indolent qui évolue en myélome multiple doit-il être traité pour éviter cette évolution et rendre cette maladie curable avec une toxicité acceptable et une qualité de vie non altérée ? L'objectif de ce traitement est également de ne pas compromettre le traitement du myélome multiple en cas de progression vers celui-ci. Les critères de diagnostic à prendre en compte sont l'absence de défaillance organique majeure, un taux de plasmocytes dans la moelle supérieur ou égal à $10 \%$ et/ou des taux d'IgM monoclonales supérieurs ou égaux à $30 \mathrm{~g} / \mathrm{dl}$ dans le sang. La progression du myélome indolent vers le myélome multiple varie en fonction du nombre de facteurs de risque :

- dix ans avec un seul facteur de risque ;

- cinq ans avec deux facteurs de risque ;

$-1,9$ an avec trois facteurs de risque.

Cela explique l'intérêt d'un traitement précoce pour ce dernier groupe à haut risque d'évolution dont la définition n'est pas clairement établie. Ces facteurs de risque sont :

- taux sérique du composant monoclonal supérieur à $30 \mathrm{~g} / \mathrm{l}$;

- infiltration médullaire supérieure à $10 \%$ de plasmocytes ;

- et IRM anormale ;

- ratio $\kappa / \lambda$ anormal (rapport des immunoglobulines légères kappa et lambda sécrétées; en cas d'anormalité de ce rapport, le pronostic de la maladie est mauvais) ;

- immunophénotypage aberrent $(>95 \%)$ : critère non valide par toutes les équipes ;

- réduction des Ig normales.

Pour le moment, toutes les recommandations sont unanimes pour ne pas traiter ces myélomes indolents en dehors des essais cliniques. Cependant, la publication des travaux de Matteo et al. en août 2013 qui évaluaient l'utilisation du lénalidomide associé à la dexaméthasone chez les patients à haut risque d'évolution pour retarder cette évolution ont a priori montré l'intérêt d'un tel traitement. En effet, le temps à progression n'est pas atteint dans le bras « traitement» vs le bras " observation » avec seulement $15 \%$ des patients qui ont progressé (vs $59 \%$ des patients dans le bras « observation »). Respectivement dans le bras " traitement » et dans le bras « observation ", la survie globale était de $93 \%$ à trois ans et de $76 \%$ à trois ans. Ces données démontrent que le traitement par REV DEX retarde la progression et prolonge la survie et semble donc avoir un intérêt, mais cette étude présente de nombreux biais méthodologiques. À ce jour, le traitement par lénalidomide ne doit pas dépasser deux ans, et l'évolution de ces patients prétraités en phase indolente n'est pas certaine. En tout état de cause, le lénalidomide n'a pas encore d'AMM dans ce cadre, et des données supplémentaires devront venir confirmer ces données. De fait, une polémique fait rage actuellement sur la définition des groupes à risque, et ces résultats sont à prendre avec la plus grande prudence. Une fois que les experts se seront accordés, nous saurons quoi faire...

\section{Leucémies à tricholeucocytes}

Nous avons également retenu une communication présentant l'intérêt du vemurafenib dans le traitement de la leucémie à 
tricholeucocytes (traitement déjà utilisé dans le traitement du mélanome métastatique). C'est une leucémie à cellules B qui représente environ $2 \%$ de tous les cas de leucémies de l'adulte. Les traitements disponibles sont :

- les interférons $\alpha$ ou $\beta$ avec des taux de réponse faible, de l'ordre de $10 \%$ (la disparition des tricholeucocytes est rarement obtenue avec ces traitements) ;

- les analogues puriques (désoxycoformycine et cladribine), avec de meilleurs résultats ( $75 \%$ de réponse complète), mais là encore, la persistance de cellules tumorales est observée malgré la disparition des tricholeucocytes.

L'une des caractéristiques posant le rationnel de l'utilisation du vemurafenib (inhibiteur de BRAF) dans le traitement de ces leucémies, est la présence de la mutation V600E de BRAF chez plus de $90 \%$ des patients. L'étude de phase II qui a été présentée incluait des patients en rechute après traitement ou en réponse partielle après traitement par des analogues puriques. Le vemurafenib a permis de montrer une action très rapide au niveau de la diminution des leucocytes et des plaquettes, à partir du deuxième jour de traitement. Les réponses complètes qui sont obtenues interviennent généralement 40 jours après le début du traitement et se caractérisent par une absence de tricholeucocytes et une normalisation des autres lignées. Les effets indésirables les plus fréquents sont la toxicité cutanée, les arthralgies et les syndromes main-pied.

\section{Syndromes myéloprolifératifs}

La place des interférons $\alpha$-2a dans la prise en charge de la polyglobulie de Vaquez et la thrombocytémie essentielle, ainsi que l'intérêt du ruxolitinib dans la myélofibrose ont été rappelés.

De nouveaux traitements avec des mécanismes d'action différents de ceux connus dans les thérapeutiques ciblées ont été brièvement présentés :

- imetelstat (inhibiteur des télémorases) dans la thrombocytémie essentielle : la télomérase est une enzyme qui permet d'ajouter aux extrémités des chromosomes des séquences télomériques, car à chaque division cellulaire, il existe une perte de 50 à 200 nucléotides. Ainsi, l'activation de la télomérase permet d'augmenter la survie de la cellule. Ce médicament a été testé chez des patients résistants ou intolérants aux traitements habituels : 92,3\% de réponse complète (normalisation du taux de plaquettes) au bout de 6,1 semaines en moyenne.

- pentraxin-2 ou PRM-51 dans la myélofibrose : c'est une protéine recombinante permettant de réguler la différenciation des monocytes. Ainsi, elle permet de diminuer la fibrose et de réduire le volume splénique.

- nutlin-3 dans la polyglobulie de Vaquez : c'est un inhibiteur de MDM2, complexe intervenant négativement dans la régulation de p53. MDM2 est une E3 ubiquitine ligase induisant la dégradation de la P53. Comme les cellules CD34+ présentent un taux élevé de MDM2, grâce à la Nutlin-3, il y a restauration de l'apoptose.

\section{Syndromes myélodysplasiques}

Il a été rappelé la nécessité de maintenir le traitement par azacitidine le plus longtemps possible, car des réponses tardives, au-delà des six premiers mois de traitement sont fréquemment retrouvées. Cette administration prolongée doit être proposée en cas de réponse complète, partielle et même d'amélioration hématologique tant qu'il n'y a pas d'évolution et que le traitement est bien toléré.

De nouveaux traitements ont été présentés ainsi que des associations de traitements :

- inhibiteurs des HDAC en association à l'azacitidine, avec des résultats décevants pour l'entinostat et prometteurs pour le vorinostat ;

- rigosertib dans les RARB-1, RARB-2 en rechute après traitement par azacitidine, inhibiteur de PI3K isoforme $\alpha$ et $\beta$ permettant d'obtenir $52 \%$ de réponse complète ;

- association lénalidomide + azacitidine (phase II).

\section{Cancers digestifs}

Dominique Dautel, Françoise De Crozals, Florence Tabary

\section{Cancers du pancréas}

Avant de s'intéresser aux thérapeutiques du cancer du pancréas, un certain nombre de communications se sont axées sur l'épidémiologie et la génomique de ces cancers. Il s'agit du neuvième-dixième cancer le plus courant dans le monde, et il représente la quatrième cause de décès liée au cancer avec une augmentation de $1,5 \%$ par an et la survie à cinq ans est de $6 \%$. Un certain nombre d'altérations génétiques sont associées à ces cancers, notamment $90 \%$ des patients présentent une mutation KRAS, mais aussi d'autres types de mutations telles que p16 CDKN2A, TP53, SMAD4/ TGF $\beta R 1+2$, BRCA 1, BRCA2...

Tout au long du processus tumoral, et notamment dans la phase précancéreuse (qui dure en moyenne 12 ans environ, les mutations génétiques - dont KRAS — sont déjà présentes). Avec les progrès de la pharmacogénomique, des scores sont maintenant établis pour déterminer la sensibilité des tumeurs à des traitements. Notamment, parmi ces marqueurs, hENT1 est un biomarqueur d'efficacité de la gemcitabine ; c'est un récepteur nucléosidique transmembranaire exprimé par la tumeur qui permet la pénétration intracellulaire de la gemcitabine ; la détermination du niveau d'expression de hENT1 permet de prévoir l'efficacité de la gemcitabine. Si le taux est élevé, la gemcitabine sera efficace ; en 
revanche, si le taux est bas, la gemcitabine sera moins efficace. L'objectif de ce type de détermination est, évidemment, de ne faire profiter des thérapeutiques que les patients qui pourraient y répondre et ne pas perdre de chance si, au contraire, le patient se présente comme « résistant » au traitement.

Parmi les études qui ont été présentées, en traitement de première ligne métastatique du cancer du pancréas non prétraité, deux études comparant la gemcitabine à de nouveaux traitements sont à souligner :

- PRODIGE 4 comparant le protocole FOLFIRINOX à la gemcitabine qui a démontré, en faveur de la triple association, une amélioration de la survie globale $(11,1$ mois vs 6,8 mois) avec un hasard ratio (HR) de 0,57 , soit une réduction du risque de décès de $43 \%$;

- MPACT comparant nab-paclitaxel associé à la gemcitabine à la gemcitabine seule, démontrant, là encore, une supériorité de l'association en termes de survie globale ( 8,5 vs 6,7 mois) avec une réduction du risque de décès de $28 \%$.

Les critères de choix entre ces deux nouvelles thérapeutiques dépendent de l'état général du patient; en effet, pour recevoir le FOLFIRINOX, le patient doit avoir un PS de 0 ou 1 et un bilan hépatique à peu près conservé. La problématique du choix entre les deux traitements ne se pose par actuellement en France, puisque le nab-paclitaxel n'est pas encore disponible, même s'il est mieux toléré.

Pour les traitements en deuxième ligne métastatique, il n'a pas été démontré d'existence d'un standard thérapeutique. La gemcitabine ou le 5-FU utilisés en deuxième ligne n'ont pas montré de bénéfice particulier, au même titre que la radiothérapie.

Fort heureusement, de nombreuses molécules sont actuellement en développement dans cette indication avec des essais en cours, notamment le ciblage des voies de l'IGR1R, de RAS, de l'EGFR, de mTOR... Peut-être l'un de ces produits permettra-t-il un jour de mieux traiter ces patients.

\section{Cancers gastriques}

Il n'y pas eu de nouveautés présentées à ce congrès au sujet des cancers de l'estomac qui se situe au quatrième rang des cancers les plus courants (deuxième cause de mortalité par cancers) avec des survies assez courtes de l'ordre de huit à dix mois. Parmi les essais présentés, il y avait tout de même l'essai TOGA, étude de phase III, qui a permis l'enregistrement du trastuzumab dans cette indication. Il s'agissait de comparer, chez des patients présentant un cancer de l'estomac surexprimant HER2 :

- capécitabine + cisplatine ;

- capécitabine + cisplatine + trastuzumab.

Les résultats de cet essai ont montré une amélioration de la survie globale dans le bras avec le trastuzumab avec
13,8 mois vs 11,1 mois, et une réduction du risque de décès de $26 \%(\mathrm{HR}=0,74)$.

D'autres études sont en cours, notamment avec le TDM1. Il s'agit d'une étude avec trois bras de traitement comparant TDM1 administré toutes les trois semaines, TDM1 administré de façon hebdomadaire et un taxane. L'analyse de cet essai est en cours. Par ailleurs, des études avec des inhibiteurs de l'EGF-R (cétuximab et panitumumab) ont, elles, été arrêtées, car négatives.

En deuxième ligne de traitement, un essai de phases I-II faisant intervenir le rilotumumab (anticorps humanisé antimet) a été présenté. Il visait à comparer le protocole de référence (épirubicine-cisplatine-Xeloda ${ }^{\circledR}[\mathrm{ECX}]$ ) associé au placebo à ce même protocole associé au rilotumumab. Les résultats ont montré que l'adjonction du rilotumumab améliorait la survie globale (11,2 vs 8,9 mois) et la survie sans progression (6,9 vs 4,6 mois). Ces résultats devront évidemment être confirmés par une étude de phase III.

Enfin, le ramucirumab (anticorps monoclonal recombinant humain de type $\operatorname{IgG} 1$, antagoniste du récepteur au VEGF2) a été utilisé dans deux essais cliniques :

- essai REGARD vs placebo avec une survie globale de 5,2 vs 3,9 mois et une réduction du risque de décès de $22,4 \%$ $(\mathrm{HR}=0,776)$;

- essai RAINBOW, en association au paclitaxel, en cours.

Les effets indésirables associés au ramucirumab sont classiquement, dans cette classe, des événements cardiovasculaires à type d'hypertension artérielle et les accidents thromboemboliques.

\section{Cancers des voies biliaires}

Les seules données présentées très brièvement concernaient la carcinogenèse des cancers des voies biliaires ; elle fait intervenir fortement l'activation de la voie des MAPKinases (activée dans $75 \%$ des cas), mais aussi les mutations KRAS qui sont retrouvées chez une forte proportion de patients (de 10 à $45 \%$ des cas). Ces données constituent le rationnel des essais qui sont conduits avec les thérapeutiques ciblées, notamment les anti-MEK, le selumetinib et le trametinib, actuellement développés en phase I et en phase II.

\section{Cancers colorectaux}

Parmi les études présentées dans le cadre de la session dédiée aux cancers colorectaux, c'est évidemment de stratégies de maintenance qu'il a été question. Trois études principales ont été retenues ici :

- essai OPTIMOX2 qui a démontré que la maintenance par LV5FU2 était efficace ;

- une étude suisse qui, après une chimiothérapie de première ligne associée au bévacizumab, visait à comparer l'efficacité d'un traitement de maintenance par bévacizumab seul à 
une abstention thérapeutique. Cette étude n'a pas démontré d'intérêt du bévacizumab en maintenance, puisque l'amélioration de la survie globale n'était pas significative ;

- au contraire, l'étude CAIRO III, de design un peu différent, a pu montrer cette différence ; dans l'étude, les patients recevaient un traitement par CAPOX (capécitabine-oxaliplatine), associé au bévacizumab. À la randomisation soit le traitement était arrêté, soit les patients recevaient une association de capécitabine et de bévacizumab ; à la première progression (PFS1), le CAPOXbévacizumab était réintroduit jusqu'à la seconde progression (PFS2).

Les données de la PFS1 ont montré une différence significative entre le bras « observation » et le bras « capécitabine + bévacizumab» $(8,5$ vs 4,1 mois $)$, ce qui démontre un intérêt de la maintenance. De même, les données de PFS2 étaient significatives, mais avec une amélioration bien moindre (11,5 vs 10,5 mois) après réintroduction du traitement initial.

En termes de «nouveautés thérapeutiques », il a été présenté un nouvel antimétabolite oral, le TAS102, association de trifluridine et de tipiracil, qui permet d'obtenir une concentration de la trifluridine au niveau cellulaire. Il a fait l'objet d'une étude de phase II, le comparant au placebo chez des patients atteints de cancer colorectal métastatique, ayant déjà reçu au moins deux lignes de traitement et randomisés selon un schéma 2:1. Les résultats ont montré une amélioration de la survie globale, passant de 6,6 mois avec le placebo à 9,0 mois avec le TAS102 et une réduction du risque de décès de $44 \%(H R=0,56)$. L'analyse en sous-groupes a, en outre, démontré un bénéfice sur la survie globale et la survie sans progression chez les patients qui présentaient une mutation de KRAS. Par ailleurs, les principaux effets indésirables décrits dans cette étude étaient à type de diarrhées, de toxicités hématologiques et de fatigue. Le développement de ce produit se poursuit, avec une étude de phase III, incluant des patients KRAS mutés.

Enfin, parmi toutes les communications autour des cancers digestifs, et en particulier des cancers colorectaux, c'est l'extension du profilage moléculaire des cancers colorectaux qui a retenu notre attention, avec notamment les mutations de la voie RAS (KRAS, NRAS), mais aussi les mutations de la voie de PI3K :

- profilage moléculaire KRAS/NRAS : il est établi que $15 \%$ des patients qui présentent un type sauvage de KRAS présentent des mutations minoritaires de RAS/NRAS et de BRAF ; il s'agit de patients qui s'ajoutent au $40 \%$ de patients présentant une mutation de KRAS. Il a été démontré, dans des études, un impact de ces mutations :

- étude PRIME comparant FOLFOX à une association FOLFOX-panitumumab qui a montré un bénéfice de l'adjonction du panitumumab uniquement dans le groupe RAS sauvage ;
- étude FIRE3 comparant FOLFIRI-bévacizumab à FOLFIRI-cétuximab chez des patients KRAS sauvages qui a démontré un bénéfice du bras FOLFIRI-cétuximab chez les patients RAS sauvage et délétère chez les patients RAS mutés ;

- ces deux études permettent d'insister sur la nécessité de réaliser une analyse complète du statut RAS (KRAS/ NRAS) des patients avant de les traiter.

- profilage moléculaire PI3K : 10 à $15 \%$ des patients présentent des mutations du gène PIK3CA. Une étude avec l'aspirine a été conduite chez des patients présentant cette mutation et elle a permis de montrer un bénéfice en termes de survie sans rechute; cette mutation semble donc être un facteur prédictif de réponse à l'aspirine.

\section{Cancers gynecologiques}

\section{Olivier Aujoulat, Florence Basuyau}

La première question qui s'est posée à l'occasion des sessions dédiées aux cancers gynécologiques, et notamment des cancers épithéliaux de l'ovaire, consistait à savoir s'il était pertinent d'utiliser des schémas hebdomadaires de paclitaxel ; a priori, le rationnel scientifique repose sur un effet antiangiogénique intéressant de cette modalité thérapeutique. Au total, une quinzaine d'études comparant l'administration du paclitaxel toutes les trois semaines à un schéma hebdomadaire en association avec un sel de platine ont été présentées. Une seule étude sur quinze, coordonnée par le JGOG, a montré des résultats très positifs dans ce contexte avec des médianes de survie globale à 100 vs 62 mois, en faveur du schéma hebdomadaire.

Il est à noter que cette étude n'a inclus que des patientes asiatiques ; en outre, ce schéma semble apporter un bénéfice plus important pour les patientes qui présentent des résidus tumoraux supérieurs à un centimètre. Ce schéma thérapeutique pourrait-il constituer un standard, pour quelles patientes et quelle en serait la dose optimale puisque ces études ont fait état de doses de paclitaxel allant de 60 à $80 \mathrm{mg} / \mathrm{m}^{2}$ ?

L'autre question qui a été soulevée lors de la session concerne l'intérêt de l'ajout du bévacizumab au traitement précédent (paclitaxel hebdomadaire), au travers de l'étude GOG-262. L'objectif de l'étude étant de mettre en évidence, ou pas, une interaction bénéfique entre le bévacizumab et le protocole paclitaxel-carboplatine. Là encore, les deux schémas d'administration du paclitaxel étaient comparés dans cette étude, dont le schéma comportait un bras avec du paclitaxel administré toutes les trois semaines et un bras où les patientes recevaient le paclitaxel de façon hebdomadaire.

Les résultats ont montré qu'il n'y a pas de différence entre les deux schémas lorsque le bévacizumab est ajouté au traitement cytotoxique ; en revanche, pour les patientes qui ne recevaient pas le bévacizumab, le bénéfice du schéma 
hebdomadaire est retrouvé, comme dans l'étude précédente. Il semble donc que l'ajout du bévacizumab à un schéma d'administration hebdomadaire du paclitaxel ne soit pas favorable.

Par ailleurs, la session visait à faire le point sur l'utilisation du bévacizumab dans le traitement du cancer de l'ovaire. Pour cela, une synthèse des différentes études réalisées dans ce cadre a été présentée :

- GOG-218 et ICON-7 en première ligne de traitement ;

- OCEANS et AURELIA en deuxième ligne de traitement.

Pour l'étude ICON-7, les bénéfices observés dans les premiers résultats publiés ont été confirmés lors du congrès 2013 de l'ESMO. Dans cette étude, en effet, le bénéfice s'exprimait surtout pour les patientes à haut risque (stade III réséqué de manière suboptimale ou stade $\mathrm{IV}$ et patientes ne pouvant pas bénéficier de chirurgie), avec une traduction de ce bénéfice en survie sans progression, mais surtout en survie globale (différences significatives) :

- survie sans progression : 16,0 vs 10,5 mois, en faveur du bras bévacizumab ;

- survie globale : 39,7 vs 30,3 mois, toujours en faveur du bras bévacizumab.

Ces données confirment celles de l'étude GOG-218 et démontrent l'intérêt du bévacizumab dans le cancer de l'ovaire. Il reste à préciser la dose optimale de bévacizumab, puisque ICON-7 utilisait des doses de $7,5 \mathrm{mg} / \mathrm{kg}$ et l'étude GOG-218 des doses de $15 \mathrm{mg} / \mathrm{kg}$.

En deuxième ligne de traitement, les deux études analysées (AURELIA : patientes résistantes aux sels de platine et OCEANS : patientes sensibles aux sels de platine) ont démontré aussi l'intérêt du bévacizumab, que ce soit pour les patientes présentant un cancer sensible ou résistant aux sels de platine. Dans les deux études, il y a une amélioration de la survie sans progression : 6,7 vs 3,4 mois pour AURELIA et 12,4 vs 8,4 mois pour OCEANS. Pour l'étude AURELIA, les données de survie globale ne semblent pas être différentes, en raison de nombreux cross-over entre les bras de traitement.

D'autres alternatives thérapeutiques ont également été présentées rapidement, notamment des inhibiteurs de tyrosine-kinase (ciblant le VEGF), pour le traitement de ces cancers gynécologiques ; il semblerait ainsi que le nintédanib apporte un bénéfice sur les patientes à bas risque, que le pazopanib soit intéressant en traitement de maintenance, que le trebananib ait également un intérêt...

Pour la chimiothérapie intrapéritonéale, l'étude présentée (GOG-172, conduite chez des patientes présentant un cancer de l'ovaire) a démontré un bénéfice en survie pour les patientes (Cochrane 2006). Ce résultat est confirmé par la très longue survie globale des patientes $\mathrm{R} 0$ dans l'étude GOG 172 (127,6 mois de survie globale). Les patientes qui en bénéficient le plus sont celles chez qui BRCA1 est faiblement surexprimé (on observe 36 mois de différence en survie globale entre celles qui reçoivent IP et IV).
Par ailleurs, lors de cette session, a été posée la question : « le carboplatine peut-il remplacer le cisplatine par voie intrapéritonéale ? ». Il est possible d'utiliser du carboplatine à la place du cisplatine à la condition d'utiliser au minimum $400 \mathrm{mg} / \mathrm{m}^{2}$ de carboplatine. En respectant cette condition, un bénéfice en survie globale est observé (51 vs 25 mois pour des patientes présentant des tumeurs de stade III ou de stade IV).

Dans le cancer du col de l'utérus à un stade avancé, le bévacizumab a été évalué au travers de l'étude GOG-240. C'est une étude de phase III, dont le schéma où les patientes étaient randomisées dans quatre bras :

- paclitaxel-carboplatine ;

- paclitaxel-carboplatine-bévacizumab ;

- paclitaxel-topotécan ;

- paclitaxel-topotécan-bévacizumab.

Il y avait donc deux groupes de patientes, à savoir :

- celles qui recevaient de la chimiothérapie seule ;

- celles qui recevaient de la chimiothérapie associée au bévacizumab.

Les résultats ont démontré que l'ajout du bévacizumab à la chimiothérapie améliorait significativement la survie globale des patientes (17,5 vs 14,3 mois dans le bras standard cisplatine-paclitaxel), mais aussi la survie sans progression et le taux de réponse. Cela dit, l'ajout du bévacizumab est également associé à des effets indésirables plus importants puisque 3 à $8 \%$ des effets indésirables de cette étude sont directement imputables au bévacizumab. Précisons néanmoins que l'amélioration de la survie globale n'a pas été accompagnée par une baisse de la qualité de vie.

L'association bévacizumab-cisplatine-paclitaxel devient un standard dans la prise en charge du cancer du col de l'utérus à un stade avancé (c'est la première thérapeutique ciblée à démontrer cela).

Quelques mots ont également été dits au sujet des traitements anti-PARP (olaparib, rucaparib, niriparib, veliparib...), mais le message à retenir de cette session est que les patientes qui bénéficient le mieux de ces thérapeutiques sont celles qui présentent une mutation de BRCA. Pour l'instant, l'avancement des travaux n'est pas encore assez poussé pour pouvoir disposer de données de survie globale ni pour pouvoir définir les meilleures associations avec ces anti-PARP.

\section{Cancers du sein}

Nicolas Cormier, Emmanuelle Serafini-Fougereau, JeanFrançois Tournamille

Parmi les sessions dédiées aux cancers du sein, de nombreuses molécules en développement ont été présentées, dans le cadre d'études in vitro, chez l'animal ou en phase précoce. Il y avait très peu de sessions « éducationnelles » au programme, finalement. 


\section{Déplétion en cuivre dans le microenvironnement des tumeurs}

Il s'agissait là, peut-être, de la présentation la plus originale de la session « sein ». Le rationnel de cette étude repose sur le fait qu'il existe une grande période de latence (plusieurs années) avant la phase métastatique ; avant l'apparition des métastases symptomatiques (diagnostic clinique de la rechute ou de la progression), il se produit deux phases bien distinctes :

- au niveau de la tumeur primitive, il y aurait une niche comportant des cellules HPC (hematopoietic progenitor cells) qui, sous l'influence de l'activation du VEGFR1, constitueraient une niche prémétastatique au niveau hépatique, avec des métastases avasculaires ;

- dans un second temps, cette niche se transforme sous l'action du VEGFR2, dans le cadre d'un switch angiogénique, avec l'apparition de macrométastases et l'apparition de cellules EPC (epithelial progenitor cells).

Il semble que le cuivre soit essentiel pour activer les médiateurs angiogéniques durant la phase prémétastatique. Le cuivre constituerait ainsi un marqueur de l'évolution tumorale, mais aussi une cible pour un chélateur du cuivre, le tétrathiomolybdate qui diminuerait l'incorporation du cuivre dans les enzymes essentielles à la synthèse des facteurs angiogéniques. Le suivi thérapeutique d'un tel traitement fait intervenir le dosage de la céruléoplasmine (méthode de dosage indirecte du cuivre plasmatique). Le tétrathiomolybdate est un traitement administré par voie orale pendant deux ans et dont l'objectif est de faire diminuer les concentrations de céruléoplasmine (suivi de l'efficacité de la chélation du cuivre). Dans l'étude qui a été présentée, 69 patientes ont été incluses et, parmi celles-ci, la quasi-totalité (94\%) présentait une tumeur de stade III/IV. Des résultats ont pu être recueillis pour 43 patientes et il s'avère que le tétrathiomolybdate est un bon chélateur du cuivre, notamment chez les patientes avec des cancers du sein triple-négatifs. La tolérance de ce traitement était bonne, malgré quelques toxicités hématologiques de grades 3-4 (sans autre toxicité de grades 3-4). Cependant, il ressort de l'étude que la corrélation entre la déplétion en cuivre et le temps de rechute/progression est très incertaine. Malgré tout, l'analyse de la survie sans progression par sousgroupes est cohérente avec les risques de rechute déjà connus. Il semble donc que la déplétion en cuivre limiterait la survenue des métastases. Il y aura peut-être d'autres informations sur cette étude au congrès de San Antonio, en décembre.

\section{Que faire en cas de « résistance » de la voie HER2 ?}

Chez des patientes qui présentent une « résistance » de la voie HER 2/3, la « sensibilité »aux traitements de cette voie peut être restaurée par l'utilisation de pertuzumab, de l'évérolimus ou des inhibiteurs de PI3K.
Le pertuzumab a été évalué dans l'étude de phase III CLEOPATRA, qui comparaît des traitements par docétaxel et trastuzumab, auquel était ajouté ou non, le pertuzumab. Dans cette étude, ayant démontré la supériorité de la triple association dans la population générale de l'étude, une analyse en sous-groupes des patientes présentant une mutation de la voie PI3K a montré un mauvais pronostic associé à cette mutation dans les deux bras de l'étude. Par ailleurs, dans l'étude de phase III BOLERO2, ayant comparé l'intérêt de l'ajout ou non de l'évérolimus à un traitement par exemestane, il a été démontré une amélioration de la survie sans progression avec 10,6 mois dans le bras évérolimus vs 4,1 mois dans le bras placebo. Enfin, d'autres études sont en cours (BKM BELLE-2 [BKM 120 et BYL 719]) ; elles font intervenir des inhibiteurs de PI3K, mais les résultats ne sont pas encore connus. D'après l'orateur de la session, dans ces situations de « résistance », il faudra probablement associer, à l'avenir, l'hormonothérapie à des inhibiteurs de la voie mTOR ou de la voie PI3K...

\section{Tamoxifène}

La présentation dédiée à l'hormonothérapie dans les traitements adjuvants du cancer du sein a permis de revenir sur les communications phares de la fin d'année dernière (San Antonio Breast Cancer Symposium 2012) et de cette année (ASCO 2013), mais aussi sur la grande question qui se pose désormais autour de la durée de traitement ; faut-il traiter les patientes cinq ans, dix ans ou à vie ? Les études ATLAS (15 000 patientes) et aTTom (7 000 patientes) ont démontré que la survie globale à 15 ans était améliorée dans ces deux études, après un traitement de dix ans :

- ATLAS : 79,8 vs $75 \%(p=0,01)$;

- aTTom : 79 vs $76 \%$ (non significatif).

Cependant, ces traitements prolongés ne sont pas dénués d'effets secondaires, notamment des événements thromboemboliques et des cancers utérins secondaires pour le tamoxifène, mais aussi une augmentation de la fréquence de l'ostéoporose, des fractures, des myalgies et arthralgies avec les antiaromatases.

Suite à la présentation de ces résultats, des schémas thérapeutiques-types ont été proposés par l'orateur (Tableau 1).

Au-delà des problématiques de survie améliorée avec des traitements prolongés, se pose la question de l'observance des patientes, notamment avec les effets indésirables gênants pour la qualité de vie... Les patientes accepteront-elles de poursuivre leur traitement pendant cinq ans supplémentaires ?

\section{Inhibiteurs de PARP}

La cible de cette classe de médicaments est la poly(ADPribose) polymérase (PARP) qui est impliquée dans la réparation de la cellule cancéreuse présentant une mutation du 
Tableau 1 Schémas thérapeutiques proposés pour le traitement par hormonothérapie.

\begin{tabular}{|lll|}
\hline & Femmes préménopausées & Femmes postménopausées \\
\hline $\mathbf{5}$ premières années & Tamoxifène & Tamoxifène ou antiaromatases \\
$\mathbf{5 - 1 0}$ ans & Continuer tamoxifène si les femmes ne sont toujours pas & Continuer le tamoxifène ou switch vers \\
& ménopausées. En cas de ménopause, poursuivre le tamoxifène & les antiaromatases \\
& ou changer de traitement (antiaromatases) & \\
& \multicolumn{1}{c}{ Choix de la patiente +++} \\
\hline
\end{tabular}

gène $B R C A$ (dans les cellules qui ne présentent pas la mutation BRCA, la réparation se fait par recombinaison homologue, via un gène suppresseur).

L'olaparib (qui serait bientôt disponible aux États-Unis) est un inhibiteur de PARP qui a fait l'objet d'une étude de dose, chez 54 patientes, comparant la dose de $100 \mathrm{mg}$ à la dose de $400 \mathrm{mg}$ (27 patientes dans chaque bras). Les résultats ont montré une meilleure survie sans progression dans le bras où la dose était la plus élevée avec 5,7 vs 3,8 mois pour le bras $100 \mathrm{mg}$. L'olaparib est soumis, comme toutes les thérapeutiques ciblées quasiment, à des mécanismes de résistance faisant intervenir les mécanismes d'efflux et les réversions de mutation du gène $B R C A$ (un gène $B R C A$ muté pourrait redevenir non muté pour résister au traitement). En termes de toxicité, aucune donnée n'a été communiquée durant ce congrès.

Des études faisant intervenir cette classe de produits sont en cours mais les résultats ne sont pas disponibles (peut-être seront-ils publiés à San Antonio, en décembre...) ; notamment :

- essai BROCADE : veliparib + témozolomide vs carboplatine + paclitaxel \pm veliparib chez des patientes présentant un cancer du sein métastatique et porteuses de la mutation BRCA ;

- essai OLYMPIAD : olaparib vs choix du praticien (capécitabine, vinorelbine ou éribuline) dans les cancers du sein métastatiques.

\section{Inhibiteurs de cyclines}

Dans la cellule normale, le complexe cycline-CDK4/6 est stabilisé par la protéine du $\mathrm{Rb}$ (rétinoblastome); la protéine $\mathrm{Rb}$ réprime le complexe cycline-CDK4/6. Dans la cellule cancéreuse $\mathrm{ER}+$, l'amplification du complexe cycline-CDK4/6, associée à une perte de $\mathrm{Rb}$, se traduit par l'entrée en mitose de la cellule. Le principe d'utilisation des inhibiteurs de cycline est de se fixer sur le complexe et, ainsi, de le stabiliser pour éviter l'entrée en mitose. Le produit qui est le chef de file de cette classe est le palbociclib, inhibiteur de CDK4/6, mais il en existe d'autres en développement actuellement en phase précoce (alvocidib, roscovitine).

C'est l'essai TRIO 18 impliquant le palbociclib qui a été le plus détaillé au cours de la session ; il s'agit d'un essai de phase I/II visant à comparer l'utilisation de létrozole associé ou non au palbociclib chez des patientes ménopausées, atteintes d'un cancer du sein ER+ à un stade avancé. Cent soixante-cinq patientes ont été incluses dans l'essai de phase II, et les résultats ont montré une amélioration de la survie sans progression avec 26,1 mois dans le bras palbociclib vs 7,5 mois dans le bras létrozole seul ; notamment, chez les patientes HER2-, les survies étaient aussi améliorées avec 18,2 vs 5,7 mois. Dans cette étude, les effets indésirables rencontrés étaient principalement des neutropénies de grade 3 (quasiment une patiente sur deux).

\section{Margetuximab}

Le margetuximab est un nouvel anticorps monoclonal chimérique, dirigé contre HER2, qui présente le même épitope que le trastuzumab et la même activité antiproliférative in vitro. Pour le moment, ce traitement n'a fait l'objet que d'une étude de phase I, qui a retenu la dose de $6 \mathrm{mg} / \mathrm{kg}$; il en est notamment ressorti, en termes de toxicité, qu'il se produisait un syndrome de relargage nécessitant une prémédication.

\section{Traitements adjuvants des patientes de bon pronostic}

La présentation a soulevé un certain nombre de questions que nous pensions résolues à ce jour, notamment :

- l'étude ECOG 2197, comparant un traitement par quatre cycles d'AC vs quatre cycles d'AT, chez des patientes présentant entre zéro et trois ganglions envahis et qui a montré une absence de différence significative à cinq ans entre les deux traitements, mise à part la toxicité ;

- la comparaison des protocoles AC et des protocoles TC chez toutes les patientes a montré que les protocoles TC étaient plus efficaces, mais plus toxiques ;

- chez 1060 patientes qui ne présentent aucun ganglion envahi, six cycles de TAC ont été comparés à six cycles de FAC et il a été démontré que l'efficacité de ces traitements était équivalente.

Outre ces réponses déjà connues, le CALBG a évalué des modalités de traitement chez 3173 patientes (étude CALBG 40101) faisant intervenir paclitaxel vs protocole AC, mais aussi l'utilisation de quatre cycles de chimiothérapie vs six 
cycles. La très grande majorité ( $94 \%$ ) des patientes ne présentait pas d'envahissement ganglionnaire et $80 \%$ des patientes étaient HER2-. Les résultats ont montré que six cycles de chimiothérapie étaient équivalents, en termes d'efficacité, à quatre cycles et que le protocole $\mathrm{AC}$ est plus efficace que le paclitaxel, même s'ils sont plus toxiques (toxicité hématologique et risques de myélodysplasies et de LAM secondaires). Même si ces traitements ne sont pas superposables avec la pratique européenne (les pratiques américaines ne reposent pas sur les mêmes standards), ils confirment l'efficacité des anthracyclines en situation adjuvante.

\section{Anticorps anti-Trop 2}

Le dernier élément que nous souhaitons rapporter ici est une présentation qui a porté sur un nouvel anticorps conjugué (pas uniquement utilisé pour les cancers du sein, mais en rechute des tumeurs solides en phase I). Il s'agit d'une immunoglobuline IgGk1, conjuguée à six molécules d'irinotécan et dirigée vers un antigène trophoblastique, exprimé dans $80 \%$ des cancers épithéliaux. Un essai de phase I (IMMU 32) a permis de tester des doses comprises entre 8 et $18 \mathrm{mg} / \mathrm{kg}$. Les résultats sont intéressants dans les cancers du sein triple-négatifs.

Il n'est pas du tout certain que ce produit arrivera au terme de son développement, mais on voit bien que ces anticorps conjugués sont une piste de recherche actuelle... et d'avenir ? (comme le TDM1, notamment, qui vient d'obtenir son AMM européenne).

\section{Cancers urologiques}

\section{Bertrand Favier, Isabelle Princet}

\section{Cancers de la prostate}

Le premier produit qui a fait l'objet d'une communication est l'orteronel, un inhibiteur de CYP17A1 qui inhibe deux enzymes impliquées dans la synthèse des stéroïdes en bloquant :

- la conversion de la prégnénolone en progestérone via la 17-alpha-hydroxylase ;

- la synthèse de la DHEA via la 20-lyase pour laquelle il a une meilleure spécificité que l'abiratérone (il en découle un impact plus faible sur la synthèse des stéroïdes et donc des doses plus faibles de prednisone administrées concomitamment).

Dans l'étude de phase II qui a été présentée, 97 patients atteints de cancer métastatique de la prostate résistant à la castration, non prétraités par chimiothérapie, ont été inclus. Quatre doses ont été évaluées, associées ou non à la prednisone $(300,400,600$ et $600 \mathrm{mg} \times 2 / \mathrm{j})$ et les résultats ont montré une diminution des taux de PSA supérieurs à $50 \%$ après 12 semaines, chez $54 \%$ des patients inclus, toutes doses confondues. Parmi les patients évaluables selon les critères RECIST (51 patients sur les 97 patients inclus), dix patients ont montré une réponse partielle et 22 patients avaient une maladie stable. En termes de tolérance, les principaux effets indésirables étaient à type de fatigue et de nausées.

Suite à cette étude de phase II, une étude de phase III (en cours : l'étude C21005) incluant 1083 patients atteints de cancer métastatique de la prostate résistant à la castration et prétraités par chimiothérapie (dont le docétaxel) a été présentée. Cette étude comportait deux bras :

- $400 \mathrm{mg}+5 \mathrm{mg}$ de prednisone (deux prises quotidiennes);

- placebo $+5 \mathrm{mg}$ de prednisone (deux prises quotidiennes).

L'analyse intermédiaire de cet essai a mis en évidence une survie globale équivalente dans les deux bras $(p=0,26)$. Cependant, l'étude se poursuit, car il semblerait que les objectifs secondaires aient montré des résultats intéressants.

Trois autres produits (TOK001, ODM201 et ARN-509) ont été présentés :

- le TOK001 est un inhibiteur de plusieurs mécanismes (inhibiteur de la CYP17-lyase et antagoniste des récepteurs aux androgènes) qui, dans des études de phase I et de phase II, a démontré sa bonne tolérance ;

- l'ODM201, évalué dans les études ARADES 1 et ARADES 2, a démontré dans trois sous-groupes de patients (patients naïfs de chimiothérapie et d'inhibiteurs du cytochrome 17, patients prétraités par chimiothérapie mais naïfs d'inhibiteurs de CYP17 et patients traités par inhibiteurs de CYP17) que le taux de réponse variait selon l'exposition ou la non-exposition à des traitements antérieurs (respectivement 65,32 et $9 \%$ ) ;

- l'ARN509 est un antagoniste des récepteurs aux androgènes qui a fait l'objet d'une étude de phase I/II et qui a démontré sa bonne tolérance malgré des effets indésirables à la dose de $240 \mathrm{mg} / \mathrm{j}$. Dans ce cadre, la diminution du taux de PSA de plus $50 \%$ à 12 semaines était mise en évidence pour $91 \%$ des patients non métastatiques, pour $88 \%$ des patients métastatiques non prétraités et de $29 \%$ chez des patients prétraités par abiratérone. Une étude de phase III est en cours, mais aucune donnée n'est disponible pour le moment.

Enfin, pour le cancer de la prostate (et comme depuis plusieurs années), il a été rapporté des données sur l'utilisation du Radium 223 qui a enfin obtenu son agrément FDA au mois de mai 2013, sous le nom de marque de Xofigo ${ }^{\circledR}$. L'essai de phase III (ALSYMPCA) avait évalué l'utilisation de six injections de Radium 223 (614 patients) vs placebo (307 patients) chez des patients présentant un cancer de la prostate et des métastases osseuses ; pour mémoire, l'utilisation du radium 223 permettait une augmentation de 3,6 mois de la survie globale (14,9 vs 11,3 mois) et une diminution du risque de décès de $30 \%$ 
environ. En outre, le délai avant la survenue des douleurs liées aux métastases osseuses était aussi augmenté à 15,6 vs 9,8 mois. En termes de tolérance, cet émetteur alpha provoque des effets indésirables à type de diarrhées et de toxicités hématologiques, globalement bien gérées. Ce traitement pourrait a priori être utilisé chez tous les patients atteints de cancer de la prostate métastatique avec des métastases osseuses; il est également actuellement évalué chez les patientes atteintes de cancer du sein.

\section{Cancers du rein}

La première communication présentait toutes les études en cours dans le cancer du rein en phase adjuvante. Trois voies thérapeutiques se dégagent de cette synthèse : la voie du VEGF, celle de mTOR et les « autres » :

- la voie du VEGF : essais ASSURE, PROTECT, SORCE, ATLAS, S-TRAC et E2810;

- la voie mTOR : essai EVEREST;

- les autres voies : essai ARISER.

Dans ces études, les principaux médicaments utilisés étaient le sorafenib, le sunitinib, le pazopanib et l'axitinib pour la voie du VEGF, l'évérolimus pour la voie mTOR et le girentuximab pour la voie « autre ». Toutes ces études représenteront plus de 7700 patients et ce sont les résultats qui en découleront qui permettront de définir la stratégie thérapeutique de prise en charge du cancer rénal en situation adjuvante.

Une autre communication portait sur le cabozantinib dans le cancer du rein et de la prostate. Il a d'abord été rappelé que le cabozantinib (XL 184) est un inhibiteur de tyrosine-kinase, double inhibiteur du VEGFR2 (bloque l'angiogenèse) et de la voie cMET, (bloque le développement des métastases et la croissance tumorale). Ce médicament est en cours d'évaluation dans le cancer de la thyroïde du rein et de la prostate. Le rationnel de son utilisation repose sur :

- l'augmentation de la sécrétion de l'HIF $1 \alpha$ aboutissant à la synthèse de VEGF et de MET dans le cancer du rein ;

- l'existence d'une surexpression du MET et du VEGF dans le cancer de la prostate.

Dans ces indications, des études ont donc été présentées avec le cabozantinib :

- cancers du rein : étude de phase I incluant 25 patients qui avaient déjà reçu au moins deux lignes de traitement. La posologie utilisée était de $140 \mathrm{mg} / \mathrm{j}$ et les résultats ont montré une survie sans progression de 14,7 mois et un taux de réponse partielle de $28 \%$ (maladie stable dans $52 \%$ des cas); en outre, ce traitement semble avoir un véritable impact sur les douleurs osseuses ;

- cancers de la prostate : étude de phase II vs placebo incluant 172 patients atteints de cancer métastatique de la prostate résistant à la castration et prétraités par chimiothérapie. Dans cet essai, le produit était utilisé à la dose de $100 \mathrm{mg} / \mathrm{j}$ et les résultats ont montré une amélioration de la survie sans progression ( 23,9 vs 5,9 mois) et une stabilisation de la maladie pour $75 \%$ des patients.

Enfin, la dernière communication traitait des nouveaux inhibiteurs de tyrosine-kinase dont le dovitinib. Ce médicament, utilisé à la dose de $500 \mathrm{mg} / \mathrm{j}$, cinq jours sur sept était comparé, dans une étude de phase III au sorafenib chez 570 patients atteints de cancer métastatique du rein, mais les résultats ont montré que la survie globale et la survie sans progression n'étaient pas significatives et que les taux de réponse étaient identiques dans les deux bras. Il est donc peu probable que ce traitement soit utilisé dans le cancer du rein dans un avenir proche. Par ailleurs, d'autres études de phase III sont en cours avec le cabozantinib et le lenvatinib, mais les résultats n'ont pas été communiqués.

\section{Cancers de la vessie}

Dans cette indication, il n'était pas question de thérapeutiques ciblées, mais bel et bien d'optimisation du protocole de chimiothérapie dans le cadre de cancer de la vessie en situation néoadjuvante, en comparant un protocole conventionnel (MVAC classique) à un protocole dit accéléré ou dose dense, permettant d'administrer les médicaments aux mêmes doses, mais avec des intervalles plus rapprochés (tous les 15 jours, sous couvert de G-CSF).

L'objectif principal de l'étude AMVAC, essai de phase II, était d'évaluer l'impact du MVAC accéléré sur le délai de prise en charge chirurgical et de préciser la tolérance d'un tel traitement. Dans cette étude, les patients recevaient donc trois cycles de MVAC accéléré, suivis d'une chirurgie à la neuvième semaine de traitement. Quarante-quatre patients ont été inclus et $38 \%$ d'entre eux ont obtenu une réponse pathologique complète avec un traitement bien toléré. L'intervention se concluait en indiquant que ce traitement permet d'obtenir un raccourcissement du délai avant chirurgie chez ces patients et qu'à l'avenir, il faudrait évaluer l'impact d'un tel schéma sur la survie.

Pour la prise en charge des patients traités dans un contexte métastatique, il a été présenté un tableau reprenant les stratégies thérapeutiques connues, sachant que, en deuxième ligne métastatique des études impliquant le bévacizumab (CALB90601) et l'OGX427 sont en cours.

\section{Cancers du poumon, cancers ORL, mélanomes}

\section{Michel Doly, Martine Duban}

\section{Cancers du poumon}

L'immunothérapie dans le cancer bronchique non à petites cellules fait partie des communications particulièrement 
intéressantes, avec notamment les inhibiteurs de PD-1 et de PDL-1. Le PD-1 (programmed death protein-1) est exprimé sur les lymphocytes $\mathrm{T}$ et empêche leur activation ; les tumeurs expriment à leur surface le ligand du PD-1, le PDL-1 ; il en ressort un intérêt puisque la liaison PD1-PDL-1, en inhibant l'activation des lymphocytes T, favorise la croissance tumorale. Il s'agit donc, pour cette thérapeutique ciblée, d'empêcher la liaison entre PD1 et son ligand.

Les inhibiteurs de PD-1 en développement sont nombreux ; parmi eux, le nivolumab est actuellement étudié dans une étude de phase III, à la dose de $3 \mathrm{mg} / \mathrm{kg}$ administré tous les 15 jours jusqu'à progression vs un traitement par docétaxel, en deuxième ou troisième ligne de traitement dans les cancers bronchiques à petites cellules et les cancers non à petites cellules. Cet essai a montré que la tolérance du nivolumab était acceptable puisque le produit est moins toxique que l'ipilimumab (anti-CLA4), car il génère moins de réponse inflammatoire. De même, le lambrolizumab (MK 3475) est en cours de développement vs le docétaxel dans ces indications.

Parmi les inhibiteurs de PDL-1, le MPDL3280A est à un stade encore plus précoce de développement (phase I). Il semble que la réponse à ce traitement soit, logiquement, fonction du statut immunohistochimique PDL-1 de la tumeur, et il apparaît qu'il est également bien toléré puisqu'il n'y a que très peu d'effets indésirables de grades 3-4.

Ces traitements sont encore en cours de développement, mais les perspectives consistent à développer des combinaisons thérapeutiques ciblant les mécanismes clés de la régulation de l'immunité, le but étant de « faire remonter les lignes » à l'immunothérapie et de la placer en amont de la prise en charge thérapeutique lorsque la masse tumorale réduite est plus facilement cernable, voire d'associer l'immunothérapie et la chimiothérapie.

Parmi les thérapeutiques ciblant des voies de signalisation, des traitements dirigés contre les mutations $A L K$ EML4 sont aussi en cours de développement. Ces mutations touchent 3 à $4 \%$ des patients; il s'agit principalement de patients jeunes, non-fumeurs, sans prépondérance ethnique et ces patients présentent souvent des adénocarcinomes de type mucineux. Dans ce cadre, le crizotinib a déjà une AMM, avec des taux de réponse importants, de l'ordre de $60 \%$ et des taux de survie sans progression supérieurs à ceux obtenus avec la chimiothérapie cytotoxique. Cependant, il existe des résistances à ces traitements, associées à la survenue de métastases cérébrales. Dans cette classe, des médicaments de deuxième génération sont en cours de développement également; il s'agit de l'alectinib, de l'AP26113 et du LDK378. Ce sont des produits qui sont actifs chez les patients naïfs ou résistants au crizotinib, avec une efficacité en particulier sur les métastases cérébrales.

Pour les patients porteurs de mutations de l'EGFR, acquises après traitement par erlotinib ou gefitinib, des produits sont développés, notamment pour la mutation p790 de l'EGFR. Dans ce cadre, une étude de phase I d'escalade de dose est en cours chez 56 patients préalablement traités par erlotinib avec le CO-1686, inhibiteur de tyrosine-kinase ciblant les formes mutées de l'EGFR. Dans cette étude, il a été observé une régression tumorale chez huit des neuf patients présentant la mutation p790. En cours d'étude, ce produit a fait l'objet d'une modification de formulation pour améliorer sa biodisponibilité et réduire sa toxicité (cutanée et digestive).

Par ailleurs, un antiangiogénique multicible, le nintédanib, ciblant le VEGFR 1-3, le PDGFR et le FGFR 1-3 a fait l'objet de plusieurs études de phase III :

- l'étude LUME-LUNG 1 : il s'agit d'un traitement de deuxième ligne, administré à raison de $200 \mathrm{mg}$, deux fois par jour en continu, comparé au placebo, en association avec le docétaxel ;

- l'étude LUME-LUNG 2 : il s'agit de la même étude conduite chez des patients atteints de cancers bronchiques non à petites cellules non épidermoïdes, en association avec le pemetrexed, cette fois-ci.

Malheureusement, ces études n'étaient pas concluantes, en raison d'un manque de ciblage des patients à traiter. Une autre étude est en cours avec ce produit, chez des patients présentant une amplification de FGFR-1.

Enfin, les inhibiteurs de RAF et de MEK sont à l'étude actuellement chez les patients porteurs de mutation BRAF V600E, notamment avec une association de vemurafenib et de trametinib (anti-MEK). Par ailleurs, la comparaison du selumetinib (anti-MEK) au docétaxel n'a pas montré de résultats satisfaisants sur la survie globale, mais un avantage significatif sur la survie sans progression.

\section{Cancers de la tête et du cou}

Actuellement, s'agissant des cancers de l'oropharynx, il est établi que plus de $80 \%$ des cas sont associés à un statut HPV + et que, quel que soit le traitement mis en œuvre, la survie globale des patients dont la sérologie HPV est positive est supérieure à celles des patients dont la sérologie est négative, avec une survie à cinq ans $30 \%$ supérieure chez les patients $\mathrm{HPV}+$. Se pose donc la question de savoir quel est le meilleur traitement pour les patients qui ont un statut $\mathrm{HPV}+$. Cela a été évalué, pendant cette réunion, au travers de deux essais thérapeutiques :

- essai E1308 : essai de diminution de dose de radiothérapie après une chimiothérapie d'induction; les résultats ne sont pas concluants ; 
- essai Quaterback : essai de diminution de dose de radiochimiothérapie après chimiothérapie d'induction ; cet essai est en cours et les résultats ne sont pas disponibles.

Par ailleurs, la diminution de la morbidité des traitements, qui passe par ces réductions de doses de radiothérapie et de chimiothérapie, passe aussi par l'utilisation des robots chirurgicaux (chirurgie transbucale, notamment). Là encore, les données relatives à l'utilisation de ces robots ne sont pas disponibles, mais on peut retenir que le pronostic des patients $\mathrm{HPV}+$ est meilleur que celui des patients HPV-, même dans les maladies à un stade avancé $(75 \%$ de patients en vie après trois ans). La conclusion est que, à ce jour, il ne faut pas réduire la thérapeutique (dose de radiothérapie, dose de chimiothérapie) en dehors des essais cliniques, en raison de l'absence de données concluantes en ce sens.

\section{Cancers de la thyroïde}

Les cancers de la thyroïde sont très majoritairement des cancers folliculaires différenciés ( $97 \%$ des cas). Pour ces cancers différenciés, le traitement repose sur la chirurgie (thyroïdectomie), la radiothérapie métabolique à l'iode 131 et le blocage de la TSH. Dans certains cas de résistance à l'iode 131, qui ne concerne que 5 à $15 \%$ des patients (dont la survie n'est que de 2,5 à 3,5 ans), il n'existe pas de traitement standard. Dans ce cadre, un essai de phase III comparant le sorafenib $(400 \mathrm{mg} \times 2 / \mathrm{j})$ au placebo (étude DECISION) chez 417 patients a montré une amélioration de la survie sans progression dans le bras sorafenib, sans amélioration de la survie globale. Suite à ces résultats, le sorafenib est en cours d'enregistrement.

Dans ce contexte, les autres études cliniques présentées étaient :

- une étude de phase I (UPCC18310) chez des patients porteurs de la mutation V600E de BRAF, naïfs de traitement ou prétraités par un inhibiteur du VEGFR2, et traités par vemurafenib : les résultats de cette étude ont montré des taux de réponse meilleurs pour les patients naïfs de tout traitement.

- une étude de phase III (ASTRA) comparant le selumetinib au placebo pendant cinq semaines, en traitement adjuvant de l'iode 131 à la dose de $100 \mathrm{mCi}$. L'objectif de cet essai est d'évaluer si la résistance à l'iode 131 pouvait être levée avec l'association au selumetinib. Cette étude est encore en cours et les résultats ne sont donc pas disponibles.

\section{Mélanomes}

Comme dans le cancer bronchique, l'immunothérapie est évaluée dans le mélanome, puisque le PDL-1 est surexprimé dans 40 à $50 \%$ des cas. Une étude évaluant l'association de l'ipilimumab (anti-CTLA4) et du nivolumab (anti-PD-1) a permis de montrer un taux de réponse objective de $40 \%$ et des régressions tumorales dans $31 \%$ des cas. Cependant, les toxicités de ces deux produits s'associent aussi et donc les effets indésirables de cette association restent importants.

Par ailleurs, trois études de phase I sont en cours avec le nivolumab, le lambrolizumab et le MPDL3280.

La toute dernière communication portait sur le mélanome de l'uvée dont la survie globale est dramatique, avec 6 à 12 mois de survie en moyenne et pour lequel aucun traitement n'existe à l'heure actuelle. Dans ce contexte, l'ipilimumab a été évalué dans une étude de phase III qui a permis de montrer un contrôle de la maladie jusqu'à trois mois. Par ailleurs, une nouvelle voie fait l'objet de recherches actuellement ; il s'agit de la mutation du gène Gnaq (gène qui code pour la sous-unité $\alpha$ de la protéine G qui est à l'origine du signal lumineux au niveau de la rétine). Chez les patients porteurs de la mutation Gnaq, le selumetinib a été évalué dans une étude de phase III vs le témozolomide ou la dacarbazine. Les résultats de cet essai ont montré une régression tumorale chez $50 \%$ des patients sous selumetinib vs $11 \%$ chez les patients sous témozolomide ou dacarbazine. Il semble cependant que la réponse au selumetinib pourrait être indépendante du statut mutationnel Gnaq.

\section{Conclusion}

Encore une fois, ce congrès a fait le point sur l'actualité en cancérologie après les congrès de début d'année (ASCO, ESMO, pour les principaux), mais aussi sur l'actualité à venir dans les congrès de fin d'année (ASH, San Antonio Breast Cancer...).

Ce point d'étape, plus que nécessaire, pour « se poser » et faire le point sur l'actualité est indispensable au pharmacien, notamment pour sa mission de garant du bon usage des traitements et de la bonne prise en charge des patients. Ces éléments, nous l'espérons, alimenteront vos discussions de $\mathrm{RCP}$ et les choix thérapeutiques à venir.

Conflits d'intérêt : Les auteurs déclarent les conflits suivant :

Olivier Aujoulat, Florence Basuyau, Nicolas Cormier, Dominique Dautel, Françoise De Crozals, Michel Doly, Martine Duban, Bertrand Favier, Isabelle MadelaineChambrin, Catherine Ollivier, Muriel Paul, Isabelle Princet, Emmanuelle Serafini Fougereau, Florence Tabary, JeanFrançois Tournamille - Prise en charge des frais d'inscription, de déplacement et d'hébergement pour le congrès Mount Sinaï 2013 par Roche. 
Jean-Baptiste Rey - Liens d'intérêt personnels (honoraires, invitations à des congrès, trois dernières années) : Amgen, Archimedes Pharma, Boehringer-Ingelheim, Eisai, IDIS, Leo Pharma, Merck Serono, MSD, Mundipharma, Pfizer, Roche, Vifor Pharma - Liens d'intérêt associatifs (montants significatifs perçus par l'association Européenne de formation pour les pharmaciens, trois dernières années) : Amgen, Boehringer-Ingelheim, Eisai, IDIS, Janssen, Leo Pharma, LFB, Mundipharma, Roche - Liens d'intérêt institutionnels (relations personnelles ou associatives avec les payeurs et les autorités de santé, trois dernières années) : ARS Champagne-Ardenne, Institut national du cancer.

Open Access : cet article est publié sous licence Creative Commons Attribution qui autorise toute utilisation, diffusion ou reproduction sous tous formats à condition que les références à l'auteur et à l'article original soient clairement mentionnées. 